\title{
Gingival bleeding reduction using a carbamide peroxide based tooth paste with lactoperoxidase.
}

\author{
Sabrina Dominguez-Moreira ${ }^{1}$, Carmen Llena-Puy ${ }^{2,3}$, Leopoldo Forner-Navarro ${ }^{3}$, Pedro Martínez-Canut ${ }^{4}$, \\ José Amengual-Lorenzo 5 \\ ${ }^{1}$ Private practice, Valencia, Spain. \\ ${ }^{2}$ Primary Care Dentist, Department 9, Valencian Health Service, Spain. \\ ${ }^{3}$ Professor, Department of Stomatology, University of Valencia, Spain. \\ ${ }^{4}$ Ex Professor, Department of Stomatology, University of Valencia. Private practice in Periodontology. Valencia, Spain. \\ ${ }^{5}$ Associate Professor, Department of Stomatology, University of Valencia, Spain
}

Correspondence:

C/ Albuixech $n^{\circ} 8$, pta 2

46021 Valencia

963604877

llena@uv.es

Dominguez Moreira S, Llena Puy C, Forner Navarro L, Martínez Canut $\mathrm{P}$, Amengual Lorenzo J. Gingival bleeding reduction using a carbamide peroxide based tooth paste with lactoperoxidase. J Clin Exp Dent. 2011;3(5):e452-5.

http://www.medicinaoral.com/odo/volumenes/v3i5/jcedv3i5p452.pdf

Article Number: 50639 http://www.medicinaoral.com/odo/indice.ht

(c) Medicina Oral S. L. C.I.F. B 96689336 - eISSN: 1989-5488

eMail: jced@jced.es

\begin{abstract}
Introduction: The combination of carbamide peroxide, thiocyanate or enzymes such as amynoglucosidase or gluco-oxidase is able to reduce gingival inflammation.

Objective: To evaluate antiseptic efficacy, in relation to reduced gingival bleeding, of a new dentifrice containing carbamide peroxide and lactorperoxidase.

Materials and method: A sample of 32 patients, with periodontal pockets of more than $3 \mathrm{~mm}$ in the vestibular area and $4 \mathrm{~mm}$ at interproximal sites, was divided into two groups of 16 and 17 patients respectively. Group A used a dentifrice containing 3\% carbamide peroxide and 5\% lactoperoxidase plus $0.022 \%$ sodium fluoride for three weeks followed by a control dentifrice with only $0.022 \%$ sodium fluoride for another three weeks. Group B used the same dentifrices but in reverse order. Efficacy was assessed by measuring the bleeding sites at the start of the study, and after application of the first and second dentifrices.

Results: After applying the toothpaste object of this study, group A showed a significant reduction in bleeding sites $(p<0.05)$ and a slight non-significant increase when the control toothpaste was applied $(p>0.05)$. After application of the control toothpaste, group B showed a significant reduction in the average number of bleeding sites and after application of the study toothpaste the group showed a greater reduction in the average number of bleeding sites, which was also significant in relation to the first phase $(p<0.05)$. Conclusions: Reduction of gingival bleeding by the carbamide peroxide based dentifrice with lactoperoxidase was found to be superior to the control fluoride dentifrice.
\end{abstract}

Key words: lactoperoxidase, carbamide peroxide, dentifrice, gingival bleeding. 


\section{Introduction}

Hydrogen peroxide and the enzymes related to its combustion, particularly peroxidases, form part of the salivary peroxidase system which maintains oral health in three ways: protecting host cells from hydrogen peroxide secreted by bacteria and defending cells by regulating the amount and controlling the toxicity of this powerful oxidising agent; inactivating carcinogens and mutagens; and regulating the amount and distribution of oral microflora by inhibiting the growth and metabolism of many bacterial species (1).

This protection mechanism is activated by interaction between hydrogen peroxide, in the oral medium, (2) with two products secreted by the salivary glands: an enzymatic one, peroxidase (3), and the thiocyanate ion $(\mathrm{SCN})(4,5)$. In the presence of hydrogen peroxide, SCN oxidation is catalysed by peroxidase to form hyopthiocyanate (OSCN). OSCN is innocuous for the host but inactivates the growth and metabolism of many pathogens and oxidises carcinogenic compounds and mutagens, transforming them into much less harmful forms (1).

OSCN is abundant in the salivary peroxidase system although there are other similar oxidised compounds and other enzymes such as catalase or dismutase peroxide with a similar function. Knowledge of this system is important, its components, peroxide, enzymes and SCN are applied with more or less success in our clinical practice as antimicrobials, dental whiteners and even to alleviate the symptoms of xerostomy (6). Thus, there is a clear need to research more deeply the formulation, effectiveness, toxicity and dose-dependency of such products and, by emulating the peroxidase system, to study the effectiveness of the peroxides- enzymes association. The catalysing action of certain enzymes can increase the effectiveness of peroxide for bleaching teeth, so lower doses are required, thereby reducing the potential toxic effect. This situation has been demonstrated in vivo on tetracycline stained teeth (7). A combination of peroxide and enzymes could also be applied to achieve the intended antimicrobial effect of peroxide and our research focuses on this aspect.

Up to 1995 very few clinical studies were published on the antiseptic activity of peroxides $(14,15,16)$ or enzymes $(8,9,19,20,21)$. Among the previous scanty contributions, the association of peroxides, enzymes and SCN is awakening a certain amount of interest and that is the reason for this present prospective, double-blind, crossover trial designed to evaluate the antiseptic and antimicrobial effectiveness, in relation to reduced gingival bleeding, of a new dentifrice containing carbamide peroxide and lactoperoxidase, initially proposed as a tooth bleaching product.

\section{Material and Methods \\ Sample characteristics}

The most uniform sample possible was chosen as regards hygiene habits and periodontal state in order to assess whether the study dentifrice could provide more benefits than a fluoride dentifrice. The sample consisted in a total of thirty-three patients who gave their informed consent to the study. Ages ranged between 21 and 69 years with an average age of $40 \pm 14$ years with a sex distribution of 23 women and 10 men.

Inclusion criteria were: a mesial probe depth of $3 \mathrm{~mm}$ or less and an interproximal depth of $4 \mathrm{~mm}$; a maximum of six missing teeth, excluding third molars; natural intact dentition in the anterior teeth area, where the clinical assessments were made, good plaque control with an initial Silness and Löe Plaque Index (10) of $<2$ in these teeth. Exclusion criteria were: fixed orthodontic treatment or removable prosthetic rehabilitation; inability to brush correctly; prior history of hypersensitivity or specific allergy to any of the products used in the study; pregnancy; drugs intake with possible gingival side effects; and chronic systemic illnesses with possible gingival effects.

\section{Experimental study}

Patients were randomly distributed in two groups: group A with 16 individuals and group B with 17. Group A used dentifrice 1, initially, for three weeks and then dentifrice 2 for the following three weeks. Group B used dentifrice 2 initially followed by dentifrice 2 (Fig. 1).

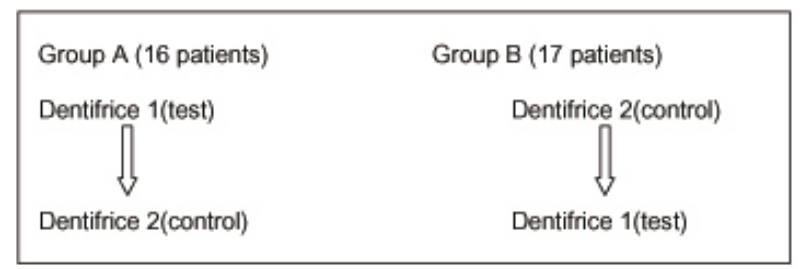

Fig. 1. Paste application

The clinical parameter chosen to reflect the possible antimicrobial capacity of the product was the Gingival Bleeding Index on probing (11). Probe pressure was standardised with a pressure-controlled probe (12). Assessments were made at three moments: in the baseline phase and at the end of the first and second phases of treatment.

Measurements were taken by a single explorer, in mesial, medial and distal areas of the vestibular aspects of the maxillary and mandibular canines and incisors (36 sites in total). Number of bleeding sites is expressed rather than the percentage, following the original index. To standardise brushing, each patient was provided with a soft toothbrush with a small rectangular head (Laboratorios Kin, Barcelona, Spain) at the start of the study and when the dentifrice was changed. They were told how to place the dentifrice on the toothbrush and the amount to use. Patients were told to brush three times a day for three minutes each time over the three weeks of 
each phase.

\section{Product composition}

The researchers did not know which dentifrice was being studied until the end of the study. Both dentifrices looked the same, except for an identification label with the numbers 1 or 2 . They were in the form of a gel and a paste which were applied to the toothbrush in equal parts. The gel for dentifrice 1 contained 3\% carbamide peroxide and the paste contained 5\% lactoperoxidase and $0.022 \%$ sodium fluoride. Dentifrice 2 (control) contained only sodium fluoride (in the same concentration as product 1) in the paste and a gel with no active components.

\section{Statistical treatment}

Average bleeding sites were compared at the start of the study and at the end of each treatment phase for both groups (ANOVA). Intra-group changes in the two phases of the study were analysed by variance analysis for matched-pair data. In both cases the confidence level was $95 \%$.

\section{Results}

Groups A and B showed no significant differences at the start of the study as regards gingival bleeding index or probe depth.

Table 1 shows the changes in average bleeding sites in each study phase for both groups. After the first phase, group A, which applied the dentifrice containing carbamide peroxide and lactoperoxidase, showed a significant reduction in average bleeding sites $(\mathrm{p}<0.05)$, whereas when the control dentifrice was applied in the second phase there was a slight, but not significant, increase $(p>0.05)$. Group B showed a significant reduction in average bleeding sites after application of the control dentifrice in the first phase; after application of the study dentifrice in the second phase, again there was a greater reduction in average bleeding sites which was also significant in relation to the first phase $(p<0.05)$.

\section{Discussion}

Hydrogen peroxide has an antiseptic effect thanks to its oxidising effect as it produces hydroxyls and other free radicals that act on a wide variety of organic compounds. However, its potential toxicity and the disparity of studies and results have meant that its antibacterial use has been sidelined. To date, studies on the application of hydrogen peroxide in this regard are scarce and they present very different experimental designs and contradictory results. Applied as a mouth rinse on an experimental gingivitis model in students (13) or by irrigation (14) the clinical results on gingivitis were positive. Subgingival irrigation with tetrapotasium peroxidiphosphate was shown to be effective in reducing plaque index and gingivitis in patients with pockets of $5 \mathrm{~mm}$ or more (15) whereas the use of a dentifrice with peroxide and sodium bicarbonate showed no significant benefit (16).

Tray-applied $10 \%$ carbamide peroxide in high caries risk patients proved effective in preventing caries and gingivitis (17).

The results of our study where carbamide peroxide is used in the form of a dentifrice at a concentration of 3\% corroborate its effectiveness in controlling gingival bleeding, the clinical expression of its antimicrobial action. As regards the use of enzymes, amyloglucosidase and gluco-oxidase in dentifrices gave discreetly positive results in relation to reducing plaque formation but did not benefit gingivitis $(18,19)$ or lacked any benefit $(20)$.

The use of a lactoperoxidase system in a dentifrice was effective at reducing S. mutans and Lactobacillus and at forming thiocyanate ions (21), in contrast to other less encouraging results $(22,23)$. Better results were obtained with the use of a fluoride dentifrice containing carbamide peroxide and thiocyanate which proved to be as effective as triclosan at reducing gingival inflammation and the formation of supragingival plaque (24).

It is not easy to obtain conclusions from an analysis of the literature. The antimicrobial effectiveness of hydrogen and carbamide peroxides is obvious and it is unanimously recognised. It appears to be confirmed by in vitro studies of oral microorganisms. It is, however, impossible to extract many conclusions on their clinical use for various reasons: firstly, due to the lack of studies, as peroxide has been rejected because of its toxic potential; and secondly because there is insufficient information on how to specify the formulation, dose and carrier for the peroxide.

The idea of combining peroxides with enzymes and $\mathrm{SCN}$ ions is promising because this combination has been anticipated in nature for maintaining oral health (the salivary peroxidase system) but there is still a long way to go before the safest and most efficient combination has been formulated. Frequent use of the term "salivary lactoperoxidase system" can lead to confusion as lactoperoxidase is similar but not comparable to human

\begin{tabular}{|l|l|l|l|}
\hline & $\begin{array}{l}\text { Average initial bleeding sites } \\
\text { (DS) }\end{array}$ & $\begin{array}{l}\text { Average bleeding sites test } \\
\text { dentifrice (DS) }\end{array}$ & $\begin{array}{l}\text { Average bleeding sites control } \\
\text { dentifrice (DS) }\end{array}$ \\
\hline Group A & $2.087(0.58)^{\mathrm{a}}$ & $1.85(0.40)^{\mathrm{a}}$ & $1.97(0.68)$ \\
\hline Group B & $2.03(0.30)^{\mathrm{b}}$ & $1.57(0.28)^{\mathrm{b}}$ & $1.81(0.34)^{\mathrm{b}}$ \\
\hline
\end{tabular}

${ }^{\mathrm{a}, \mathrm{b}}$ significant differences between groups 
salivary peroxidase. Lactoperoxidase is found in cows' milk and explains the antibacterial capacity of raw milk observed decades ago $(25,26)$. It would seem logical to seek formulations containing salivary peroxidase and other components of this system.

Few studies evaluated the clinical effectiveness of adding enzymes such as peroxidases to carbamide peroxide-based compounds, and some of them have only focused on dental bleaching (6), however, current knowledge of the action of these enzymes suggests that they fulfil two essential functions. Firstly, they enable the use of lower concentrations of peroxides as their presence optimises the liberation of active components, and, secondly, they protect living tissue in the oral cavity exposed to peroxide action.

Our results suggest that a dentifrice with very low concentrations of carbamide peroxide and lactoperoxidase is more effective at reducing gingival bleeding than a conventional fluoride dentifrice, even though the sample consisted of periodontally healthy patients.

We consider these results justify extending the research line on the antimicrobial effectiveness of hydrogen or carbamide peroxide and in particular the combination of peroxides and enzymes to increase peroxide effectiveness, enabling a reduction in the dose and the potentially toxic effects.

\section{Conclusions}

These results and the crossover design of the experiment show that the dentifrice object of our study (containing carbamide peroxide and lactoperoxidase) is clearly superior to the control fluoride dentifrice and clinically effective at reducing gingival bleeding, thereby indicating its antimicrobial capacity.

\section{References}

1. Tenovuo J, Pruitt KM. Relationship of the human salivary peroxidase system to oral health. J Oral Pathol.1984;13:573-84

2. Jago GR, Morrison M. Anti-streptococcal activity of lactoperoxidase. Proc Soc Exp Biol Med. 1962;111:585-8

3. Slowey RR, Eidelman S, Klebanoff SJ. Antibacterial activity of the purified peroxide from human parotid saliva. J Bacteriol.1968;96:575-9

4. Marshall VM, Reiter B Comparison of the antibacterial activity of the hypothiocyanite anion towards Streptococcus lactis and Escherichia coli. J Gen Microbiol. 1980;120:513-6.

5. Oram JD, Reiter B. The inhibition of streptococci by lactoperoxidase, thiocyanate and hydrogen peroxide. The oxidation of thiocyanate and the nature of the inhibitory compound. Biochem J. 1966;100:382-8.

6. Kirstilä V, Lenander-Lumikari M, Tenovuo J. Effects of oral hygiene products containing lactoperoxidase, lysozyme, and lactoferrin on the composition on whole saliva and on subjective oral symptoms in patients with xerostomia. Acta Odontol Scand. 1966; $54: 391-7$

7. Gimeno I, Riutord P, Tauler P, Tur JA, Pons A. The whitening effect of enzymatic bleaching on tetracycline. J Dent. 2008; 36: 795-800

8. Etemadzadeh H, Ainamo J, Murtomaa H. Plaque growth-inhibiting effects of an abrasive fluoride-clorhexidine toothpaste and a fluoride toothpaste containing oxidative enzymes. J Clin Periodontol
1985; 12: 607-16

9. Moran J, Addy M, Newcombe R. Comparison of the effect of toothpastes containing enzymes or antimicrobial compounds with a conventional fluoride toothpaste on the development of plaque and gingivitis. J Clin Periodontol 1989; 16: 295-9

10. Silness J, Löe H. Periodontal disease in pregnancy. II. Correlation between oral hygiene and periodontal condition. Acta Odontol Scan. 1964; 22:121-35

11. Ainamo J, Bay I. Problems and proposals for recording gingivitis and plaque. Int Dent J. 1975; 25:229-35

12. Bergenholtz A, al-Harbi N, al-Hummayani FM, Anton P, al Kahta$\mathrm{ni}$ S. The accuracy of a Vivacare true pressure-sensitive periodontal probe system in terms of probing force. J Clin Periodontol. 2000; 27: $93-8$

13. Reddy J, Salkin LM. The effect of urea peroxide rinse on dental plaque and gingivitis. J Periodontol.1976; 47: 607-10

14. Wennström J, Lindhe J. Effect of hydrogen peroxide on developing plaque and gingivitis in man. J Clin Periodontol.1979; 6:115-30

15. Listgarten MA, Grossberg D, Schwimer C, Vito A, Gaffar A. Effect of subgingival irrigation with tetrapotassium peroxydiphosphate on scaled and untreated periodontal pockets. J Periodontol.1989;60:411.

16. Taller SH. The effect of baking soda / hydrogen peroxide dentifrice (Mentadent) and a $0,12 \%$ percent clorhexidine gluconato mouthrinse (Peridex) in reducing gingival bleeding. JNJ Dent Assoc. 1993; 64: 23-5.

17. Lazarchick DA, Haywood VB. Use of tray-applied 10 percent carbamide peroxide gel for improving oral health in patients with special-care needs. J Am Dent Assoc. 2010; 141: 639-46.

18. Rotgans J, Hoogendoorn $\mathrm{H}$. The effect of tooth brushing with a toothpaste containing amylogluucosidase and glucose oxidase on plaque accumulation and gingivitis. Caries Res. 1979; 13: 144-9

19. Midda M, Cooksey MW. Clinical uses of an enzyme-containing dentifrice. J Clin Periodontol. 1986; 13: 950-6

20. Afseth J, Rölla G. Clinical experiments with a toothpaste containing amyloglucosidase and glucose oxidase. Caries Res.1983;17:472-5.

21. Jyoti S, Shashikiran ND, Reddy VV. Effect of lactoperoxidase system containing toothpaste on cariogenic bacteria in children with early childhood caries. J Clin Pediatr Dent. 2009; 33: 299-303

22. Lenander-Lumikari M, Tenovuo J, Mikola H.Effects of a lactoperoxidase system-containing toothpaste on levels of hypothiocyanite and bacteria in saliva. Caries Res. 1993;27:285-91

23. Kirstilä V, Lenander-Lumikari M, Tenovuo J. Effects of lactoperoxidase- system-containing toothpaste on dental plaque and whole saliva in vivo. Acta Odontol Scand. 1994;52:346-53

24. Rosin M, Kramer A, Bradtke D, Richter G, Kocher T. The effect of a $\mathrm{SCN}-\mathrm{H} 2 \mathrm{O} 2$ toothpaste compared to a commercially available triclosan-containing toothpaste on oral hygiene and gingival health -- a 6-month home-use study. J Clin Periodontol 2002; 29:108691

25. Cankaya M, Sişecioğlu M, Bariş O, Güllüce $M$, Ozdemir H. Effects of bovine milk lactoperoxidase system on some bacteria. Prikl Biokhim Mikrobiol. 2010;46:64-8

26. Kanwar JR, Kanwar RK, Sun X, Punj V, Matta H, Morley SM, et al. Molecular and biotechnological advances in milk proteins in relation to human health. Curr Protein Pept Sci. 2009;10:308-38. 